\title{
Shadow Lane Robust Detection by Image Signal Local Reconstruction
}

\author{
Du Mingfang ${ }^{1,2}$, Wang Junzheng ${ }^{2}, \mathrm{Li} \mathrm{Nan}^{2}$ and Li Duoyang ${ }^{2}$ \\ ${ }^{1}$ School of Automation, Beijing Union University, Beijing, 100101, P.R. China \\ ${ }^{2}$ State Key Laboratory of Complex System Intelligent Control and Decision, \\ Beijing Institute of Technology, Beijing, 100081, P.R. China \\ ${ }^{3}$ Beijing Key Laboratory of Information Service Engineering, 100101, P.R. China \\ 1314310@163.com
}

\begin{abstract}
In order to resolve shadow interference and slow image processing speed in the visual navigation of unmanned vehicles on city roads, a new lane detection algorithm based on the Inverse Perspective Mapping(IPM) of vertical sub-image reconstruction using local bands was proposed. The lane's IPM aerial image was obtained using the projective transformation of three-dimensional images of city roads. The ROI portion of the IPM map was decomposed and analyzed using the sym 3 wavelet, and after analyzing and comparing the experimental results the first and second levels of the vertical sub images were selected for the reconstruction, compression, and removal of shadows from the original image. The Canny algorithm was proposed and adopted in order to extract the edge features of the reconstructed images according to real-time road image quality. A modified, polar angle, constraint-based, fast Hough transform was used to locate the candidate lanes. Finally, the main control point, straight-line-fitting algorithm was used to fit the final lanes, which achieved the precise location and recognition of the lane lines. The California Polytechnic lane data set, a public data platform, which is now widely used in road visual recognition fields around the world, was selected for the testing and verification of the algorithm. The results of the experiment and actual operation indicated that, despite having a storage space of approximately one tenth the size of similar algorithms, this detection algorithm effectively solves the issue of shadow removal, meets the real-time requirements of roadway image processing systems for unmanned vehicles with a recognition time of less than $20 \mathrm{~ms}$, and is robust.
\end{abstract}

Keywords: Lane detection, shadow interference, Wavelet Transform, local reconstruction

\section{Introduction}

Due to their valuable military applications, unmanned ground vehicles have been studied by developed countries since the 1960s. In the early 1980s, under the support of DARPA (US), universities, such as CMU, Stanford and MIT, began researching unmanned ground vehicles. Since 2004, DARPA has sponsored the annual Desert Robotics Challenge. Based on statistics, the immaturity of the environmental perception system, not malfunctions, is the main hindrance of the practical application of unmanned vehicles $^{[1][2]}$. So far, these technical problems have not been effectively resolved, and completely independent unmanned vehicles have not been developed.

Shadows are the most common interference on outdoor roads, particularly in areas where shadows are thick and irregular. In these areas, the contrast between lane lines and road surfaces in the shadows is very low while the contrast between the interior and exterior of the shadows is high; this causes significant difficulties in feature extraction and image segmentation. Due to these circumstances, conventional image detection 
methods do not work, significantly affecting the speed of the vehicle, and even causing the loss of navigation and the vehicle to halt. Typical road images with shadow interference are shown in Figure 1.

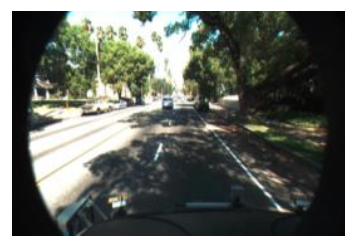

(a) Frame F00083

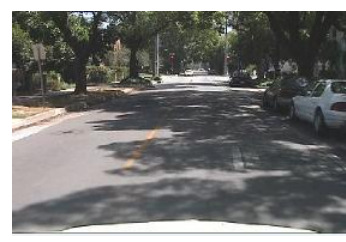

(b) Frame 1030

\section{Figure 1. Typical Road Images under Shadow Interference from California Institute of Technology}

A lot of road detection methods for shadow interference have been proposed [3-9]. Researchers developed different methods such as estimator [5], texture analysis[8] to detect or remove shadows. However, no method has been able to completely remove the effect of shadow interference on lane line recognition. In this study, a new method based on Multi resolution analysis in wavelet domain and Inverse Perspective Mapping (IPM) for removing shadow interference in road image detection is suggested, in which only the vertical sub images of a decomposed road image are selected, and the key local frequency bands of the sub images are used to reconstruct the original image. The final result is obtained by fusing the sub images between two levels, increasing the robustness of detection and recognition. This method preserves enough lane line information while removing shadow interference. Since only a portion of the frequency bands are used to reconstruct the images, they are significantly compressed; this plays an important role in increasing the speed of navigation.

\section{Overall Algorithm}

If the edge feature of the extraction is directly applied to the original image (front view), a large number of non-target edges outside the lane line targets, which cause interference in lane line detection, would be extracted, including trees, the sky, traffic signs, pedestrians, etc. The use of a Hough transform in lane line detection proves problematic since the Hough transform is intended for "clean" objects without background interference, and actual roadways do not exhibit such ideal conditions. Likewise, the direct extraction of the edges of shadowed road images would generate results including the shadows' edges, interfering with lane line detection. This problem could almost be eradicated by extracting the edges of preselected road regions on IPMs, drastically reducing interference points. This is because the shapes of the road regions from aerial perspectives are regular and easy to segment, have parallel lane lines, and are clearly defined. Therefore, in this study, we adopted IPM for lane line detection. First, the wavelet analysis was used to decompose an image into low-frequency vertical, horizontal, and diagonal sub-images. Then, the vertical sub image was selected to reconstruct the original IPM image according to lane line direction, achieving shadow removal and image compression. Through this process, the interference on the road surfaces was avoided, creating ideal conditions for the application of the Hough transform. This fast and robust method for the detection of lane lines is shown in Figure 2 . 


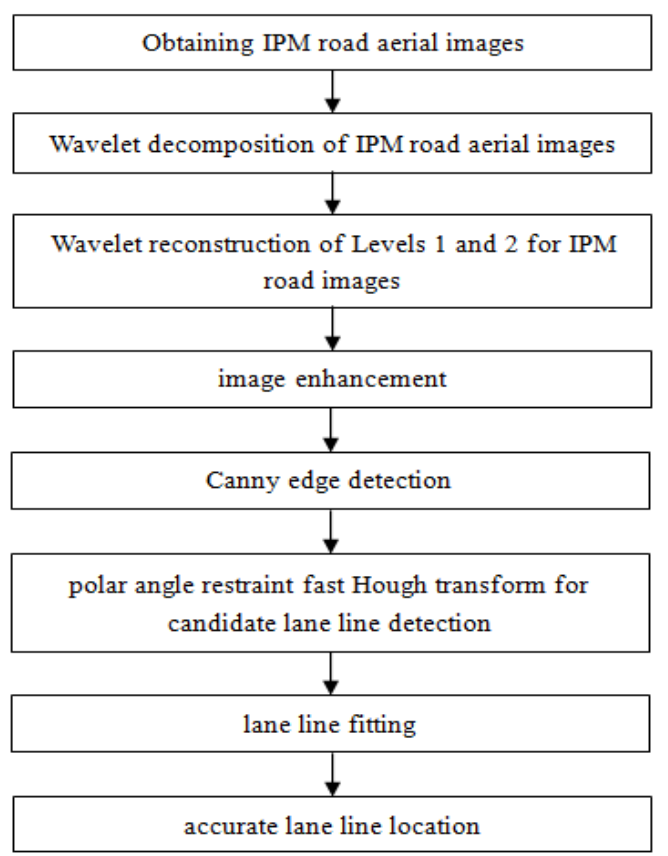

\section{Figure 2. Flowchart of the Lane Line Detection Algorithm under Shadow Interference}

These fitted lane lines could be used for vehicle positioning, lane departure warning, and lane tracking, accomplishing the visual navigation of autonomous vehicles.

\section{Obtaining IPM Aerial Views}

The perspective mapping from a three-dimensional road scene to a two-dimensional image plane brings disadvantages to the ensuing edge extraction and lane line modeling. Inverse perspective mapping uses the angle, height and position of a camera to establish a three-dimensional coordinate system in order to eliminate the perspective effect and generate the aerial image of a scene. After applying IPM, the original lane lines, which tended to intersect before, became parallel from an aerial perspective, and were easier to detect. In IPM, the typical formula, deduced by M. Bertozzi et. al., [10, 11], can be successfully applied to GOLD autonomous vehicles [2], and is written as

$$
\begin{aligned}
& x(u, v)=h \times \cot \left[(\theta-\alpha)+u \times \frac{2 \alpha}{m-1}\right] \times \cos \left[(\gamma-\beta)+v \times \frac{2 \beta}{n-1}\right]+l \\
& y(u, v)=h \times \cot \left[(\theta-\alpha)+u \times \frac{2 \alpha}{m-1}\right] \times \sin \left[(\gamma-\beta)+v \times \frac{2 \beta}{n-1}\right]+d
\end{aligned}
$$

From Eq. . (1), that the relationship between the road surface coordinates $(x, y, 0)$ in the world coordinate system and the image coordinates $(u, v)$ are derived as

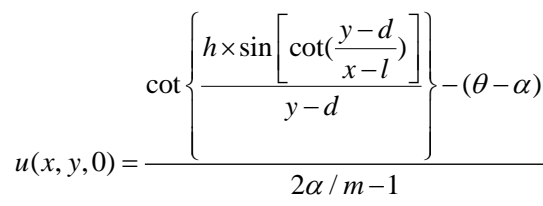

$v(x, y, 0)=\frac{\cot \left(\frac{y-d}{x-l}\right)-(\gamma-\beta)}{2 \beta / n-1}$ 
The derivation of these engineering formulae are shown in Fig. 3, where

- Coordinates of the camera in world coordinate system are $(l, d, h)$ meters,

- Resolution of the camera is $m \times n$ pixels,

- Field of view is $2 \alpha \times 2 \beta$ radians,

- Yaw angle is $\gamma$ radians, and

- Pitch angle is $\theta$ radians.

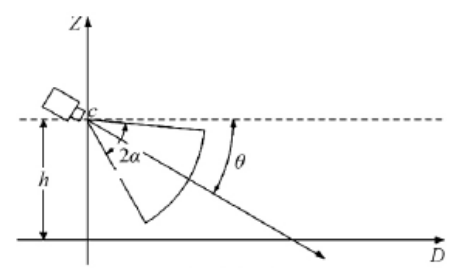

(a) Slide view

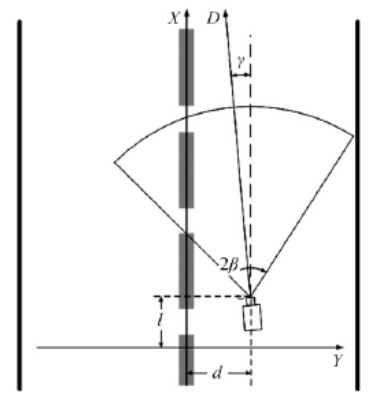

(b) Slide view

Figure 3. Location Parameters of the Camera

The relationship between the coordinates of a point $Q(x, y, z)$ in the world coordinate system and its image $q(u, v)$ is given by:

$$
\begin{aligned}
& Z\left[\begin{array}{l}
u \\
v \\
1
\end{array}\right]=\left[\begin{array}{ccc}
\frac{1}{d_{x}} & 0 & u_{0} \\
0 & \frac{1}{d_{y}} & v_{0} \\
0 & 0 & 1
\end{array}\right]\left[\begin{array}{llll}
f & 0 & 0 & 0 \\
0 & f & 0 & 0 \\
0 & 0 & 1 & 0
\end{array}\right]\left[\begin{array}{cc}
\mathbf{R} & \mathbf{t} \\
0^{\mathrm{T}} & 1
\end{array}\right]\left[\begin{array}{c}
x \\
y \\
z \\
1
\end{array}\right] \\
& =\left[\begin{array}{cccc}
a_{x} & 0 & u_{0} & 0 \\
0 & a_{y} & v_{0} & 0 \\
0 & 0 & 1 & 0
\end{array}\right]\left[\begin{array}{ll}
\mathbf{R} & \mathbf{t} \\
0^{\mathrm{T}} & 1
\end{array}\right]\left[\begin{array}{c}
x \\
y \\
z \\
1
\end{array}\right]=\mathbf{M}_{t} \mathbf{M}_{0}\left[\begin{array}{c}
x \\
y \\
z \\
1
\end{array}\right]=\mathbf{M}\left[\begin{array}{c}
x \\
y \\
z \\
1
\end{array}\right]
\end{aligned}
$$

where

- $Z$ is the normalization coefficient;

- $u_{0}, v_{0}$ are the coordinates of the central point of the image;

- $d_{x}, d_{y}$ are the horizontal and vertical physical pixel sizes of the sensor, respectively;

- $\quad f$ is the lens focal length, and $a_{x}=f / d_{x}, a_{y}=f / d_{y}, 0^{\mathrm{T}}=(0,0,0)$; 
- vector $\mathbf{t}$ is the relative positional relationship between the origins of the camera coordinate system and the world coordinate system;

- matrix $\mathbf{R}$ is the pose relationship between the axes of the camera coordinate system and the world coordinate system, which is called the camera rotation matrix;

- $\mathbf{M}_{t}$ is the inner parameter matrix, which is determined once the camera is given;

- $\mathbf{M}_{0}$ is the outer parameter matrix; and

- $\mathbf{M}$ is the projection matrix.

If the camera coordinate system and the world coordinate system coincide, the outer parameter matrix, $\mathbf{M}_{0}$, is only dependent on the rotation matrix, $\mathbf{R}$. In the pinhole perspective projection model, if the road is assumed to be flat on the surface, denoted with $z=0$, the conversion from the world coordinates of a scene point $(x, y, z)$ to the projection coordinates in the image coordinate system of this point is given by

$x=\frac{\beta h\left(u-u_{0}\right)}{\alpha\left[\left(v-v_{0}\right) \sin \theta+\beta \cos \theta\right]}$

$y=-\frac{h\left(v-v_{0}\right)}{\left(v-v_{0}\right) \sin \theta \cos \theta+\beta \cos \theta \cos \theta}$

By projecting the lanes in the image coordinate system onto the road's world coordinate system, the real lanes of roads could be obtained. The obtained lane equations of myopic and hyperopic area region by inverse projection are

$$
\begin{aligned}
& x=\frac{\left(\left(k v_{0}-u_{0}+b\right) \sin \theta-k \beta \cos \theta\right)}{\alpha} y_{w}+\frac{h\left(k v_{0}-u_{0}+b\right)}{\alpha \cos \theta} \\
& x=k_{1} \frac{(-y \beta \cos \theta)^{3}}{\alpha\left(y \sin \theta+\frac{h}{\cos \theta}\right)^{2}}+k_{2} \frac{(-y \beta \cos \theta)^{2}}{\alpha\left(y \sin \theta+\frac{h}{\cos \theta}\right)} \\
& -k_{3} \frac{y \beta \cos \theta}{\alpha}+k_{4} \frac{y \sin \theta+\frac{h}{\cos \theta}}{\alpha}
\end{aligned}
$$

Once the projection matrix was obtained, the following steps were used to perform the IPM transform of the image:

(1) Visual navigation requires a real-time process. Therefore, only the road's area of interest in front of a vehicle $(2 \mathrm{~m} \times 2 \mathrm{~m}$, with a resolution of $1 \mathrm{~cm}$ and a $200 \times 200$ grid $)$ was selected and used for the IPM transform. In the world coordinate system, a cell within the grid corresponds to $1 \mathrm{~cm} 2$ of road area, or a pixel of the IPM transformed image. The resolution of the IPM transformed image was $200 \times 200$ pixels, and only this part of the IPM's aerial view was preprocessed.

(2) For each central point in the square cell of the grid in the world coordinate system, Eq. (1) was used to calculate the corresponding image coordinates in the image coordinate system.

(3) According to the image coordinates, values were assigned to the IPM transformed image pixel by pixel.

By applying these three steps, the aerial view of a road could be obtained, as shown in Figure 4. Compared to the original image, the clarity of the lane lines in the aerial view was obvious. In fact, the view of the entire road was so clear that the manual segmentation of the road area would be possible, facilitating detection. 


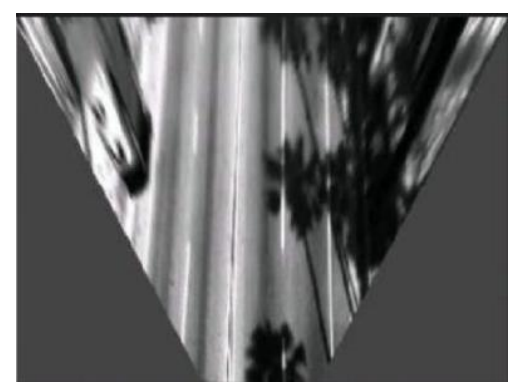

Figure 4. IPM of the Road Image under Shadow Interference

\section{Experiments and Analysis}

The California Polytechnic lane data set is a public data platform widely used in the research and validation of lane line detection efficiency around the world. This data set was selected as the experimental platform for this study.

\subsection{Experiment on IPM image obtainment}

The pseudo code for the IPM parameter setting is as follows:

camera. $\mathrm{m}=480 ; \%$ number of rows in the original image (height)

camera. $n=640 ; \%$ number of columns in the original image (width)

camera.h $=1.5 ; \%$ height of camera from the ground $(\mathrm{m})$

camera.alphaTot $=30 *$ pi $/ 180 ; \%$ half of the camera angle

camera.theta $0=-0.01373524283527 ; \%$ camera pitch angle

The installation height of the camera has a direct impact on the furthest distance that can be captured by the camera. As an example, in the front view, as shown in Figure 1, experiments with different installation heights were performed; these results are shown in Figure 5.

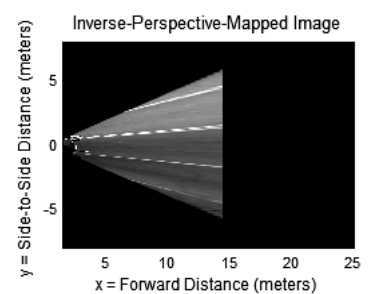

(a) $h=0.5 m$

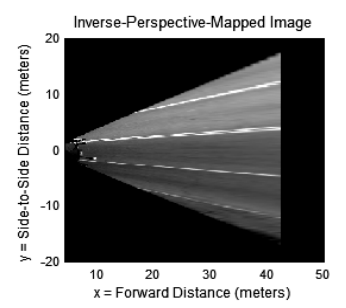

(b) $h=1.5 \mathrm{~m}$

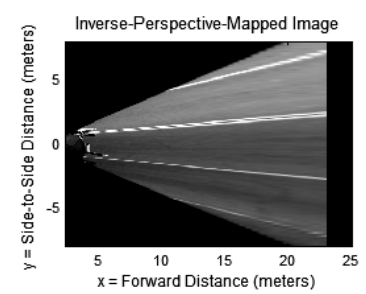

(b) $h=0.8 m$

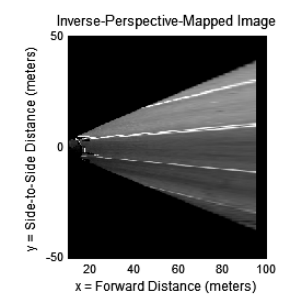

(d) $h=3.3 m$

Figure 5. Different IPM Images at Different Camera Installation Heights 
Corresponding IPM grids are shown in Figure 6.

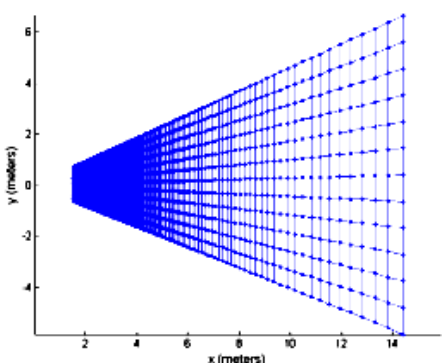

(a) $\mathrm{h}=0.5 \mathrm{~m}$

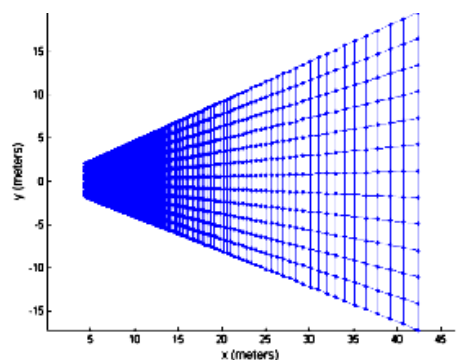

(c) $\mathrm{h}=1.5 \mathrm{~m}$

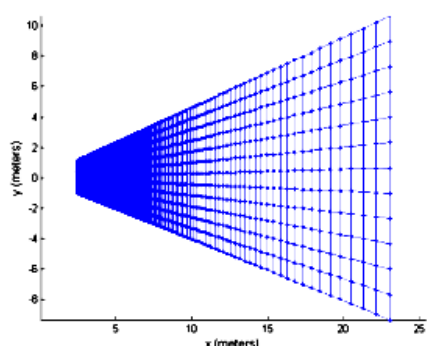

(b) $\mathrm{h}=0.8 \mathrm{~m}$

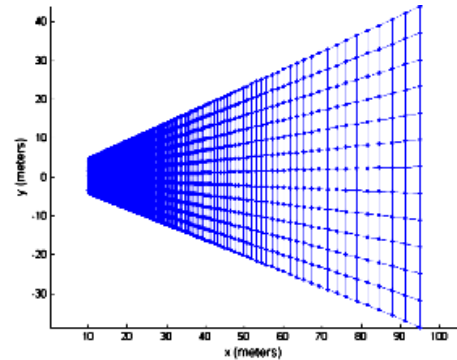

(d) $\mathrm{h}=3.3 \mathrm{~m}$

Figure 6. IPM Grids

\subsection{Image Decomposition}

From the decomposed sub images, it is apparent that the lane lines are clearest in the vertical detail sub-image, while they are very weak and almost indiscernible in other directions. Therefore, the vertical detail coefficients at each level contained the most information of the lane lines, and the sub-images in other directions could be omitted during reconstruction in order to compress the images. The vertical detail coefficient images, reconstructed images, and images reconstructed using low frequency sub-bands are shown in Figure 7.

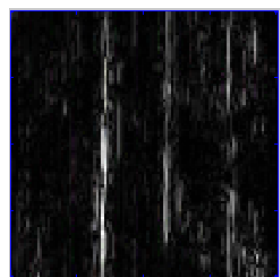

(a)

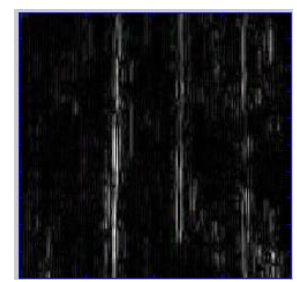

(d)

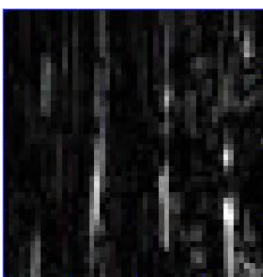

(b)

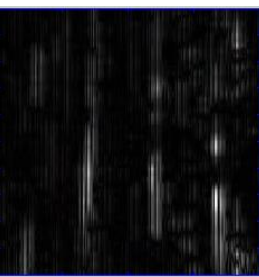

(e)

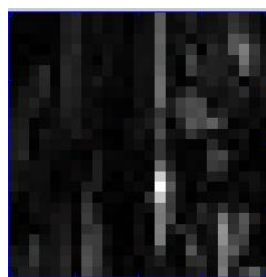

(c)

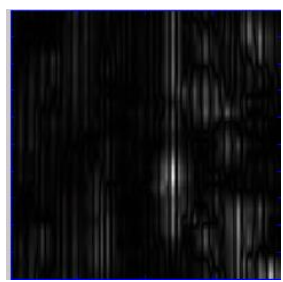

(f) 


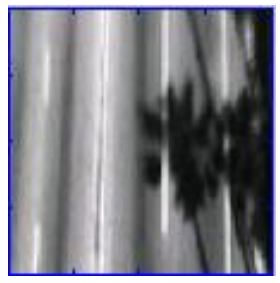

(g)

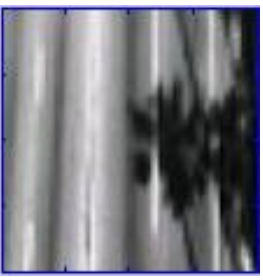

(h)

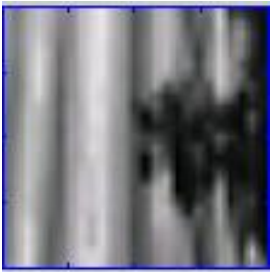

(i)

Figure 7. (a), (b), and (c) are the vertical detail sub-band images of levels 1 3; (d), (e), and (f) are the reconstructed images using the vertical detail sub-band images of levels 1 3; (g), (h), and (i) are the reconstructed images using the low frequency sub-bands of levels $1 \sim 3$.

As seen in the above experimental results, the low frequency sub-images would be suitable for reconstructing the original images. In particular, the sub image at level two could be selected for the reconstruction of the original image even though the resolution decreases somewhat; it would also be suitable for visual navigation, a highly demanded facet of real-time processing. However, the low frequency sub-image also reflected all of the elements in the original image, including the primary interference source, the shade of the trees; this poses difficulties for the ensuing lane line detection. When high frequency, detailed sub-images were used, the reconstructed images did not face this caveat. The experiments show that, when the vertical detail sub-images of levels one and two were used, the reconstructed images clearly reflected the positions of the lane lines, with the complete removal of shade interference. When the vertical detail sub-images of level two were used, the lane lines of the reconstructed original image were of especially high visual quality. . The lane lines obtained using levels higher than three were not recognizable, so these levels were discarded during the reconstruction of images. With this information, experiments on properly selected, reconstructed images were performed.

\subsection{Image Enhancement Preprocessing}

The purpose of image enhancement is to strengthen some information while weakening or removing some unnecessary information in the images. Some specific image enhancement techniques include image contrast enhancement, intensity enhancement, and contour enhancement. Different images require different enhancement processes; thus, the selection of suitable enhancement methods based on image applications is necessary. For lane IPM images, the vertical edge information needs enhancement, while the irrelevant information that needs weakening lies in other directions. The wavelet image enhancement method, due to its flexibility, meets this demand. Once the wavelet image decomposition was completed, the vertical, horizontal and diagonal coefficients were separately processed, and the coefficients of a certain portion could be controlled according to different requirements, thereby strengthening lane lines and weakening interferences. By applying the two-level wavelet decomposition to an IPM road image and manipulating the obtained coefficients, the image enhancement result, shown in Figure 8, was attained. 


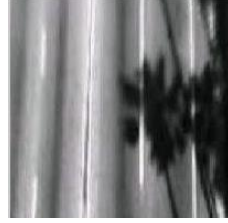

(a)

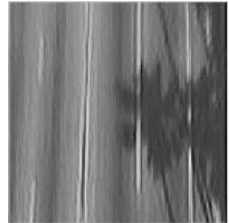

(d)

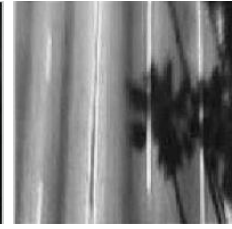

(b)

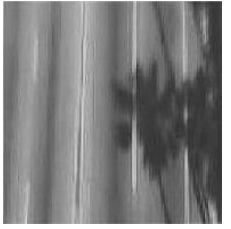

(c)

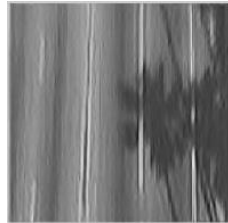

(e)

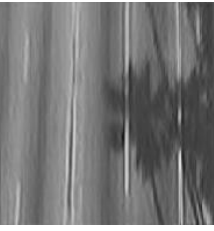

(f)

Figure 8. Results of the Wavelet Image Enhancement of an IPM Road Image

(a) Original IPM image;

(b) Global enhancement of contour and weakening details;

(c) Increasing the vertical coefficients three times at level 1 after decomposition;

(d) Increasing the vertical coefficients three times at levels 1 and 2 after decomposition;

(e) Increasing the vertical coefficients three times at levels 1 and 2 after decomposition, and decreasing the horizontal coefficients ten times;

(f)Only the vertical coefficients are preserved after decomposition.

The experimental results show that, after image enhancement, the shadows became insignificant while the lane lines were highlighted, creating advantages for edge extraction and positioning for lane lines. Gray threshold was then used to segment the image, and, finally, the lane lines were extracted. These results are shown in Figure 9.

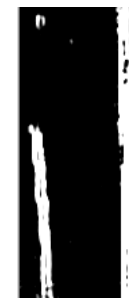

(a)

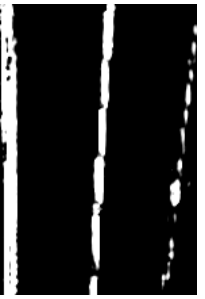

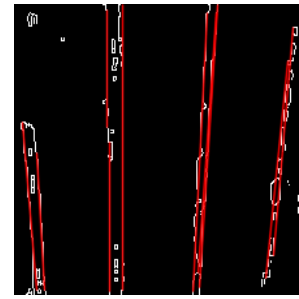

(b)

Figure 9. Lane Line Extraction Results after Enhancement of IPM Image

(a) Lane line image after enhancement

(b) Lane lines extracted by Hough transform

\subsection{Lane Detection}

The experimental results of the candidate lane line detection based on the original IPM image and the enhanced IPM image are shown in Figure 10.

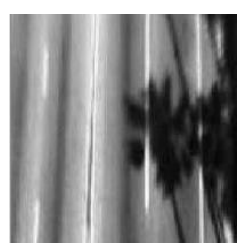

(a)

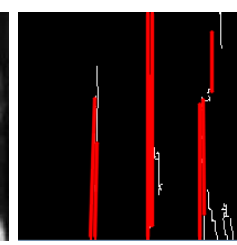

(b)

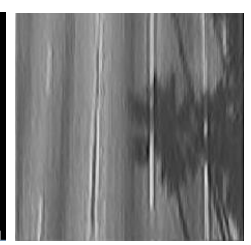

(c)

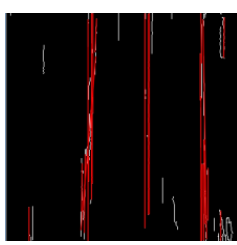

(d)

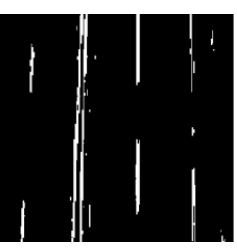

(e)

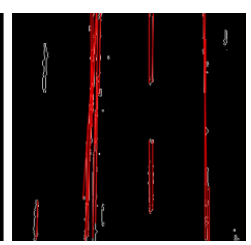

(f) 


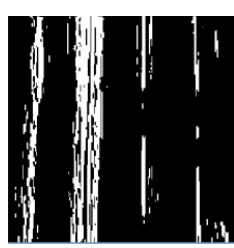

(i)

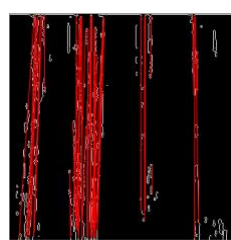

(j)

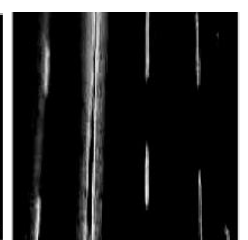

(g)

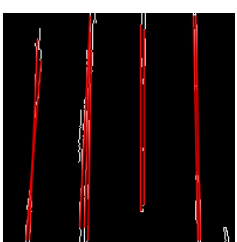

(h)

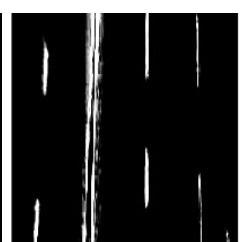

(k)

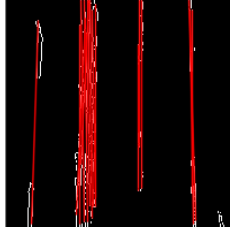

(l)

Figure 10. Candidate Lane Line Detection Results of IPM Road Image

(a) Original image

(b)Detection result using the Hough transform, with missed detections

(c) Wavelet-enhanced image

(d)Detection result using the Hough transform after wavelet enhancement

(e) Segmented image using a relatively high threshold

(f)Detection result using the Hough transform on the segmented image with the high threshold

(g)Segmented image using a relatively low threshold

(h)Detection result using the Hough transform on the segmented image with the low threshold

(i)Preprocessed image using an intensity of -99 and a contrast of 68

(j)Detection result using the Hough transform on the preprocessed image with an intensity of -99 and a contrast of 68

(k)Preprocessed image using an intensity of -98 and a contrast of 90

(1)Detection result using Hough transform on the preprocessed image with intensity of -98 and contrast of 90

The image enhancement process was applied to the compressed IPM images, which were reconstructed using vertical sub-images. The Canny operator was then used to extract the edge features. Finally, the polar angle, constraint-based, fast Hough transform was used to detect the lane lines. The final results are shown in Figure 11.

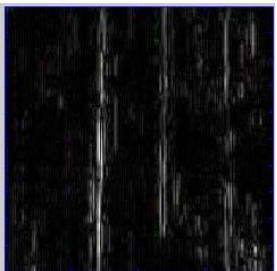

(a)

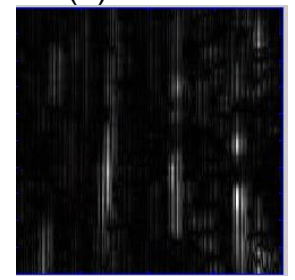

(e)

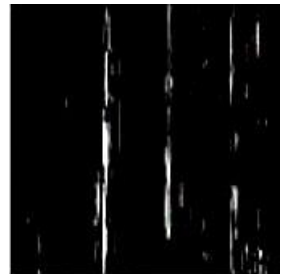

(b)

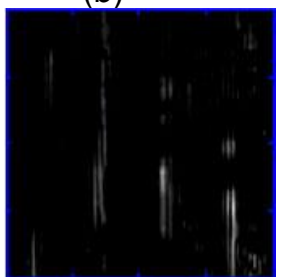

(f)

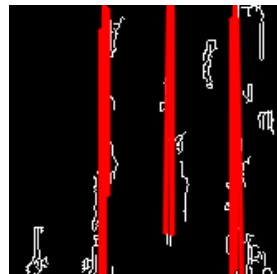

(c)

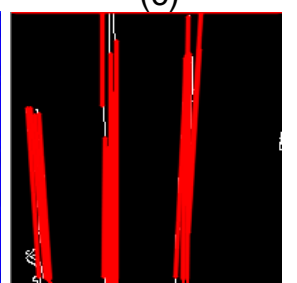

(g)

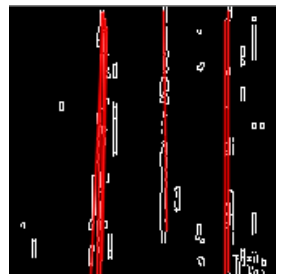

(d)

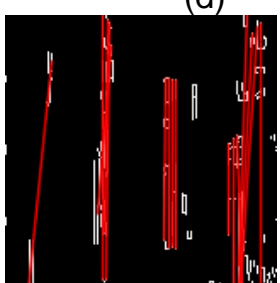

(h)

Figure 11. Lane Line Detection Result based on Sub-Image Reconstruction 
(a) Reconstructed IPM image of one level

(b) Reconstructed IPM image of one level after contrast enhancement

(c) Detection result of the reconstructed IPM image of one level using the Hough transform

(d) Detection result of the reconstructed IPM image of one level with contrast enhancement using the Hough transform

(e) Reconstructed IPM image of two levels

(f) Reconstructed IPM image of two levels after contrast enhancement

(g) Detection result of the reconstructed IPM image of two levels using the Hough transform

(h) Detection result of the reconstructed IPM image of two levels with contrast enhancement using the Hough transform

The analysis of the experimental results is as follows: (1) The Hough transforms would be able to accurately locate the lane lines on the processed IPM images; however, there would be a small number of missed detections. Two methods could be used to solve this problem: (a) the application of an image enhancement before the Hough transforms; however, the effect of this method is limited. (b) The fusion of the reconstructed images of levels 1 and 2, which could generate more robust results. This is because, as indicated by the experimental results, the missed detections of the reconstructed images at levels 1 and 2 are uncertain, but complementary to each other. (c) The location of the lane lines using the reconstructed IPM image of level 1 and the original IPM image are the same, suggesting that the detection performance of the compressed images reconstructed by vertical detail sub-images is the same as the detection performance of the uncompressed images. This provides a new, effective method for real-time degree improvement of visual navigation systems. This method is summarized as the "lane line detection method based on vertical sub-IPM image reconstruction and hierarchical image fusion". The advantages of the image are:

(1) Only two sub-images were selected to reconstruct the original IPM image, allowing for the compression of the original image without the loss of object information; this also yielded a considerable compression ratio.

(2) Shadow interference could be completely removed, and the removal effect would be better than that of other shadow processing methods;

(3) The reconstructed IPM image is binary, with a simple background and highlighted lane lines. A simple threshold segmentation method or an image contrast enhancement method could be used to remove background interference, generating an IPM image containing only lane lines and their neighborhoods, the expected ideal condition for the use of the Hough transform.

(4) Two-level, sub-IPM image fusion is adopted (the 3rd level of the sub-image could be added when necessary), which could improve the detection robustness and generate more reliable lane line detection results compared to the results using the original images.

The lane line points extracted above exist in a certain parameter subspace. Therefore, the extracted lane line points needed to be fitted in order to obtain the model parameter, and thereby locate the lane lines.

The function Polynomial was selected for fitting the lane lines. The fitting results for lane lines 1, 2, 3 and 4 are shown in Figure 12 (a). Thus, the final, accurate position of the lane lines were obtained by fitting multiple candidate lane lines. In order to comparison, taking a detection result using the ordinary method as example which is shown in Figure 12 (b). 


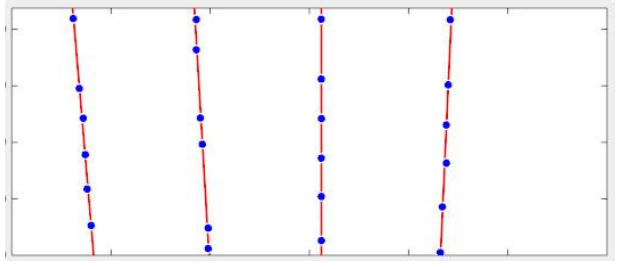

(a) Detection result using ordinary method

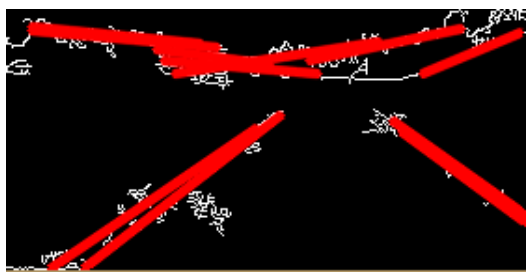

(b) Detection result using this paper's method

\section{Figure 12. Detection Result Comparison}

Figure 12 (b) indicates if the line were detected at the front view road The comparison detection results indicates that the method proposed in this paper is better than the traditional.

An experiment was performed on 100 frames of road images with shadows in order to test the performance of the proposed algorithm. The average detection time consumption is shown in Table 1.

Table 1. Experimental Result of the Performance Parameter of Lane Line Detection for Road Image with Shadows

\begin{tabular}{|c|c|c|c|}
\hline Image type & $\begin{array}{c}\text { Avg. } \\
\text { time } \\
(\mathrm{ms})\end{array}$ & $\begin{array}{c}\text { miss } \\
\text { detectio } \\
\mathrm{n} \\
\text { rate } \\
(\%)\end{array}$ & $\begin{array}{c}\text { locating } \\
\text { accuracy }\end{array}$ \\
\hline $\begin{array}{c}\text { I: original IPM images } \\
\text { II: enhanced IPM images }\end{array}$ & 10 & 2 & relatively good \\
\hline $\begin{array}{c}\text { III: compressed IPM images reconstructed by } \\
\text { vertical sub-images }\end{array}$ & 3.3 & 1 & good \\
\hline $\begin{array}{c}\text { IV: } 2 \text { level fused image of the compressed } \\
\text { IPM images, reconstructed by vertical sub- } \\
\text { images }\end{array}$ & 4.5 & 0.5 & optimal \\
\hline
\end{tabular}

Table 1 shows that the comprehensive performance of the proposed method performs significantly better than the other methods.

\section{Conclusions}

In this study, a new lane line detection method for unmanned vehicles that could effectively remove shadows was proposed. The application of multi-resolution analysis resolved the issue of strong interference in accurate lane line detection, while effectively increasing the real-time degree. The main contribution of this research lies in that it is a kind of method to solve the problem fundamentally which solves he outdoor road detection problem under the random shadow disturbance from the angle of image signal processing while the algorithm processing speed is improved substantially. The optimal comprehensive indicators of detection accuracy and speed can be achieved which cannot be found in the past works. So this study is a new invention in lane line detection field based on multiresolution image analysis technology. The application of this method will solve the uncertainty difficulties in unmanned vehicle environment perception system around the world, and play a key role in the promotion of unmanned vehicle's practical process.

In future work, the application of compressive sensing theory in wavelet domain on this system should be researched in order to further improve the real-time degree of this 
system. With the development of new theories and the application of rising technologies, a solution to the problem that visual navigation poses on the use of unmanned vehicles could be found, and unmanned vehicles could be of practical use.

\section{Acknowledgements}

Beijing Municipal Education Science and Technology Plan General Project (Grant No. SQKM201311417010); Open Project of Beijing Key Laboratory of Information Service Engineering; Beijing Union University Traffic Engineering Teaching Innovation Team Project (Grant No. BPHR2014F02).

\section{References}

[1] A. Broggi, M. Bertozzi, A. Fascioli, C. Guarino, L. Bianco and A. Piazzi, "Visual perception of obstacles and vehicles for platooning", IEEE Transaction on Intelligent Transportation Systems, vol. 1, no. 20, (2000).

[2] M. Bertozzi, "GOLD: a parallel real-time stereo vision system for generic obstacle and lane detection", IEEE Transactions on Image Processing, vol. 7, no. 11, (1998).

[3] J. Stauder, R. Mech and J. Ostermann, "Detection of Moving Cast Shadows for Object Segmentation, IEEE Transactions on Multimedia, vol. 1, no. 2, (1999).

[4] A. Bevilacqua, "Effective Shadow Detection in Traffic Monitoring Applications", Journal of WSCG, vol. 11 , no. 3, (2003).

[5] A. Prati, I. Mikic and M. M. Trivedi, "Detecting moving shadows: Algorithms and evaluation", IEEE Transactions on Pattern Analysis and Machine Intelligence, vol. 25, no. 5 (2003).

[6] J. Choi, Y. J. Yoo and J. Y. Choi, "Adaptive shadow estimator for removing shadow of moving object", Computer Vision and Image Understanding, (2010), pp. 114, 12.

[7] J. Choi, H. J. Chang and Y. J. Yoo, "Robust moving object detection against fast illumination change" Computer Vision and Image Understanding, vol. 116, no. 3, (2012).

[8] A. Leone, C. Distante and F. Buccolieri, "Shadow detection for moving objects based on texture analysis", Pattern Recognition, vol. 40, no. 22, (2006).

[9] A. Sanin, C. Sanderson and B. C. Lovell, "Shadow detection: A survey and comparative evaluation of recent methods", Pattern Recognition, vol. 45, no. 84, (2012).

[10] M. Bertozz, A. Broggi and A. Fascioli, "Stereo inverse perspective mapping: theory and applications", Image and Vision Computing, vol. 16, no. 5, (1998).

[11] S. Tan, , J. Dale, A. Anderson and A. Johnston, "Inverse perspective mapping and optic flow: A calibration method and a quantitative analysis", Image and Vision Computing, vol. 24, no. 15, (2006).

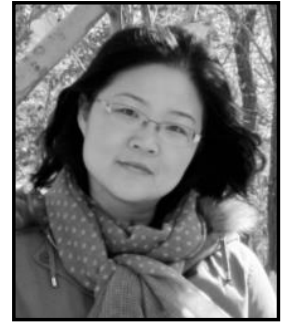

Mingfang Du, She received her Doctor Degree from Automation School of Beijing Institute of Technology and the State Key Laboratory of Complex System Intelligent Control and Decision, Associated Professor of Beijing Union University. Her main research interests include environmental perception and the control of autonomous moving robots, image based detection, image understanding.

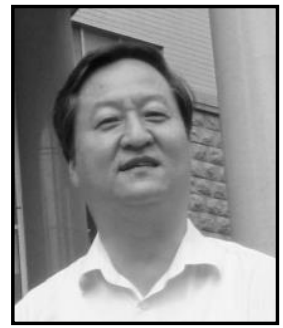

Junzheng Wang, $\mathrm{He}$ is a Professor of Automation School, Beijing Institute of Technology, Doctor, dean of the College, second director of the State Key Laboratory of Complex System Intelligent Control and Decision. His main research interests include robot and motor driving and control.

E-Mail: wangjz@bit.edu.cn 
International Journal of Signal Processing, Image Processing and Pattern Recognition Vol.9, No.3 (2016) 